\title{
NON-LINEARITY IN EXPERIMENTAL JOURNAL BEARING DYNAMICS - A NEW APPROACH
}

\author{
Wale, G. D., and Mba, D. \\ School of Engineering, Cranfield University, Bedfordshire, United Kingdom
}

\begin{abstract}
This Paper considers the effects of non-linearity in the dynamic properties of a journal bearing and presents a method of making the calculations needed in the identification of a non-linear dynamic model of the oil film. The new calculation method, together with its background and development is described and illustrated. The Method uses frequency-domain descriptions of the test waveforms and can accommodate any reasonable oil film model. It can also be used to predict orbits. It has been applied to both theoretical and experimental results and a robust procedure based on the new calculation method for the extraction of higher-order dynamic oil film coefficients is proposed and described.
\end{abstract}

Key words Journal bearings, dynamics, non-linearity, experimental methods, calculation methods

\section{Nomenclature}

$\begin{array}{ll}\text { Axx, Axy etc } & \text { Dynamic oil film coefficient } \\ \text { c } & \text { Bearing radial clearance } \\ \text { Fx etc } & \text { Force in the x-direction etc } \\ \mathrm{W} & \text { Bearing static load } \\ \mathrm{x}, \mathrm{y} & \text { Displacements in the x, y directions } \\ \omega & \text { Excitation frequency } \\ \Omega & \text { Shaft rotational frequency }\end{array}$

\section{Introduction}

It is conventional to describe the dynamic properties of a journal bearing in terms of eight first-order coefficients in a linear model. It is widely known that the relationships between the dynamic forces and dynamic displacements are not linear, except at small perturbations. A continuing problem has been how to interpret "small". The range of validity of a conventional linear model (maximum orbit size for 
which the model makes acceptable predictions) is surprisingly restricted and depends on the orbit shape [1] and the test frequency, as well as the design and running conditions of the bearing. A greater range of validity and higher accuracy may be obtained by the use of a more complete oil film model with a greater number of terms better able to describe the non-linearity.

This Paper considers the problems of non-linearity and the calculations needed to deal with a higher-order dynamic oil film model. It also describes applications to existing theoretical and experimental results and proposes a methodology for acquiring the experimental data and making the calculations needed to identify a higher-order dynamic oil film model.

\section{Higher-order dynamic oil film models}

In order to obtain a more general description of the oil film dynamics, giving a greater range of validity, it is necessary to use a mathematical model containing higher-order terms. In this Paper, the oil film coefficients are denoted in the form " $A$ " with subscripts. The first subscript indicates the line of action of the force arising, for example " $A x$ ". The next subscripts denote the parameters to be used in conjunction with this coefficient. This will use one or more of $x, y, \dot{x}, \dot{y}$. A first order (linear) coefficient uses one of the parameters at a time. An example would be $A x \dot{y}$ defining a coefficient describing a force in the $\mathrm{x}$ direction proportional to the velocity in the $\mathrm{y}$ direction. There are four first-order $A x$ force coefficients and four first-order $A y$ force coefficients, giving the familiar eight coefficients. A second order coefficient uses two of these parameters multiplied together. An example would be Axx $\dot{x}$, describing a force in the $x$ direction arising from the product of $x$ and $\dot{x}$.

A second order model will contain eight first order coefficients, eight coefficients in which the parameters are squared (sometimes called the direct terms) and 12 coefficients in which different parameters are multiplied together. This gives 28 coefficients. Other models that might be useful are:

a) The conventional first order (linear) model with 8 coefficients.

b) A model containing the first order terms and the second-order direct terms, giving 16 coefficients. 
c) A model containing the first order terms, the second order direct terms and the third order direct terms, giving 24 coefficients.

d) A model containing the first order terms and the second-order terms, giving 28 coefficients.

e) A model containing the first order terms, the second order terms and the third order direct terms, giving 36 coefficients.

All these models have been described in the Literature [2], [3], [4] and some authors have suggested that some of the terms might be omitted without much loss of accuracy. However, the extent to which the different coefficients contribute to the total force depends on the circumstances, so each situation has to be considered separately. Even higher-order models are possible, but have not been used, mostly because of the size of the resulting model and the amount of information needed to identify all the coefficients. A full third order model contains 68 coefficients, a full fourth order model contains 138 coefficients. It is evident that the smallest possible model that gives the required range of validity should be used. It will be shown later that inclusion of higher-order direct terms in the proposed model has considerable practical advantages, outweighing the disadvantage of increased model size.

\section{An overview of non-linearity in the oil film dynamics}

Non-linearity causes effects not seen in linear systems to arise, some of which may not be well known. An oil film is somewhat more complicated than many non-linear systems because of the number of factors involved. These include the effects of velocity as well as displacement in each of the two axes directions, with cross coupling between axes, displacements and velocities. It is useful to start by considering a very simplified analysis, in order to illustrate some of the problems that need to be overcome. This analysis considers only the self-stiffness in one axis. The relationship between the force and the displacement may be represented as a power series, of the form:

$F_{x}=A_{x x * x}+A_{x x^{2 *} x^{2}+} A_{x x^{3 *} x^{3}}+--\cdot---$

It is important to note that the force is expressed as a function of a power series of the displacement, rather than the other way round. However, in dynamic tests, it is 
conventional to provide excitation by specified forces, rather than by specified displacements. This complicates matters considerably, as will be explained below. A representative example, using figures from Bannister [2] gives

$F x=1.17 x+4.5 x^{2}$

Both $F x$ and $x$ are non-dimensional, $F x$ is in comparison with the static load and $x$ is in comparison with the bearing clearance. Fig 1 shows the transfer function between $F x$ and $x$ as a curve, up to displacements of $\pm 10 \%$ c. This displacement range is considered by many to be in the acceptably linear region. The linear relationship $(F x=1.17 x)$ is also shown and it will be noticed that the two are only reasonably close up to about $2 \%$ of bearing clearance. The end of range force one way is about twice that in the other.

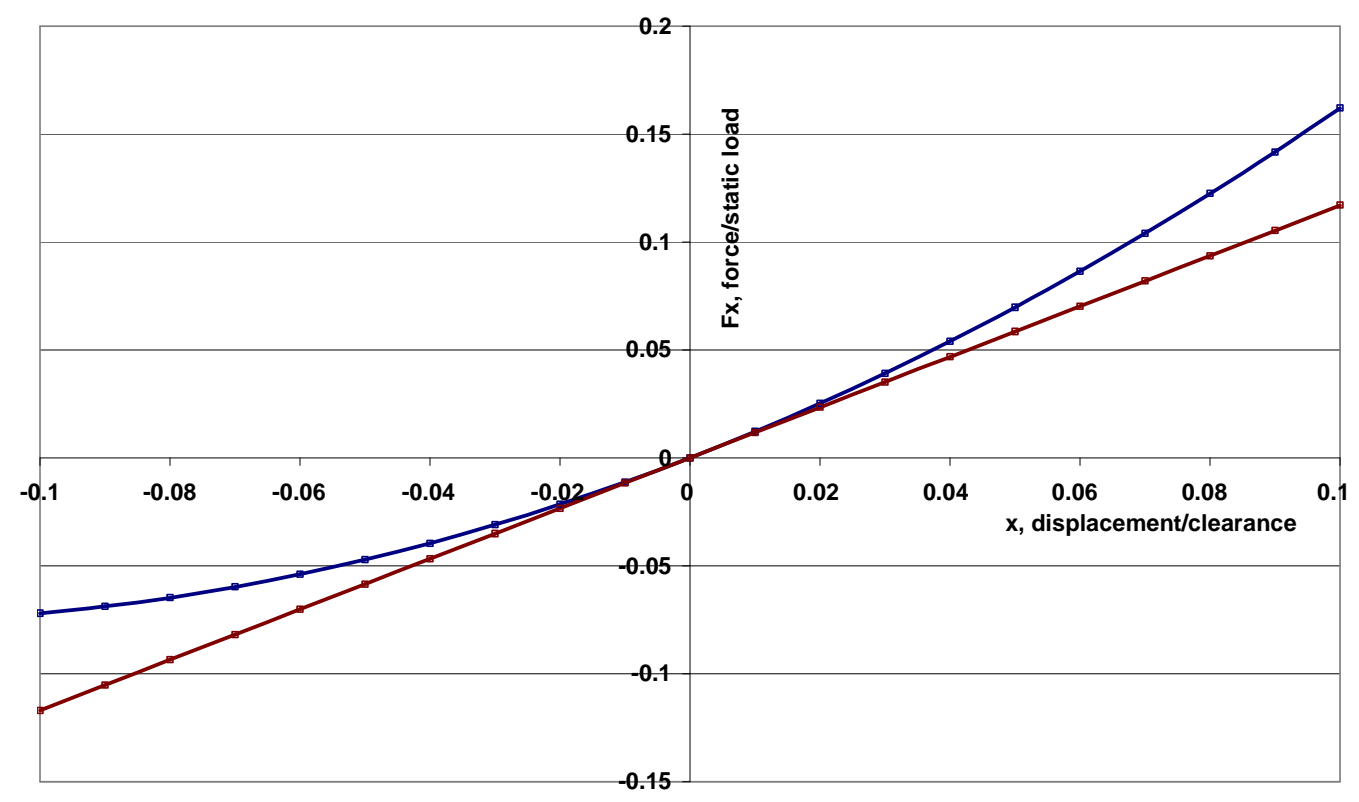

Figure 1 Transfer function between force $F_{x}$ and displacement, $x$

The response of the non-linear transfer function (see equation 2) to a sinusoidal excitation force of non-dimensional value 0.070 (0-peak) is shown in Fig 2., in which the excitation is also shown for reference. The response was calculated by solving the quadratic equation relating displacement and excitation force (Equation 2) for a succession of time steps of the sinusoidal force. Although the response is well within the $\pm 10 \%$ c range often assumed to be linear, it will be noticed that the displacement 
waveform is not sinusoidal. The positive peaks of the displacement are less than would arise in a linear system: the negative peaks are greater.

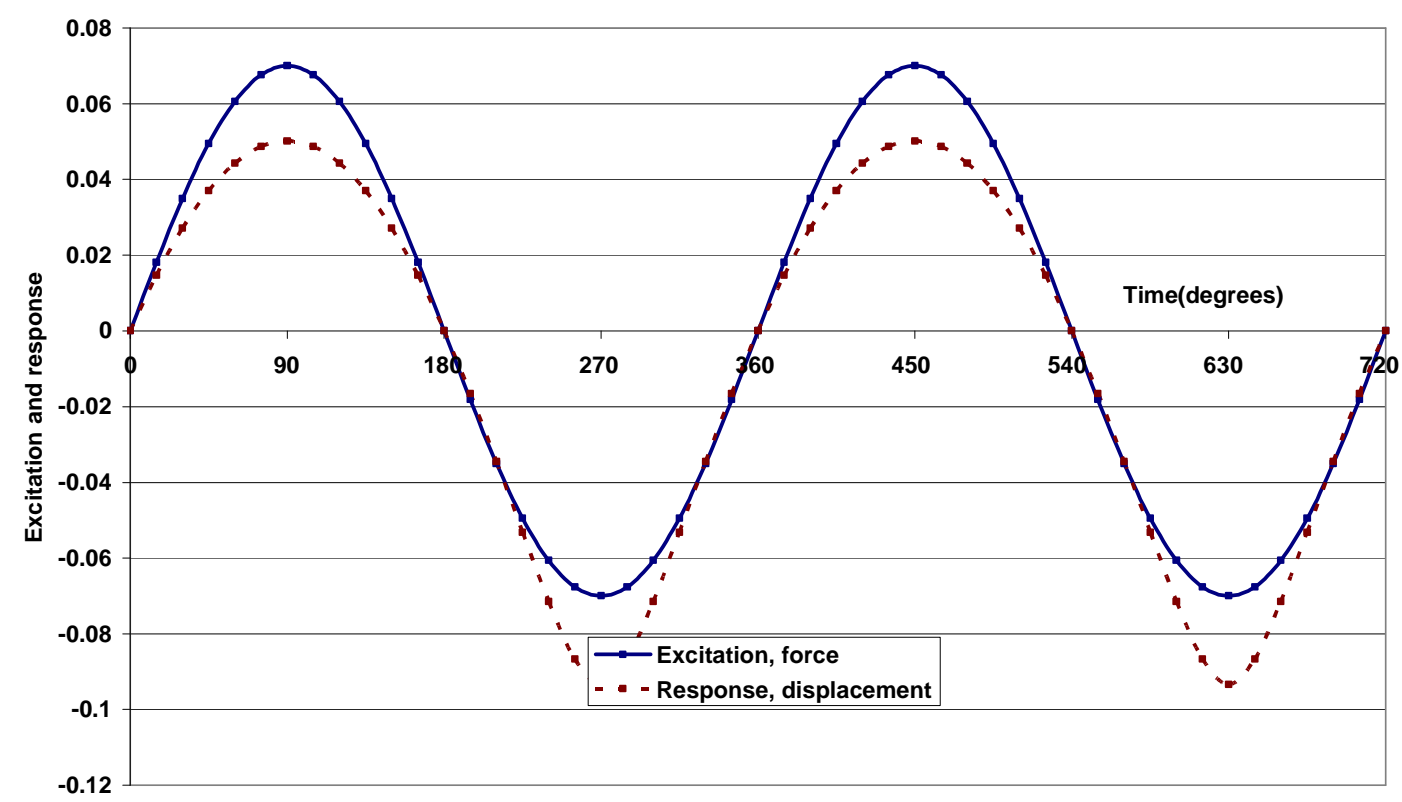

Figure 2 Displacement under sinusoidal force excitation

Both the force and displacement are periodic, so may be represented by Fourier series. On analysis, it is found that the Fourier series for displacement is infinite, although the non-linearity is only second order. Part of the spectrum is illustrated in Fig 3, with the values shown as percent of the fundamental (first harmonic). The non-dimensional value of the first harmonic is 0.0676 . The higher terms decrease in size quite quickly in this example. 


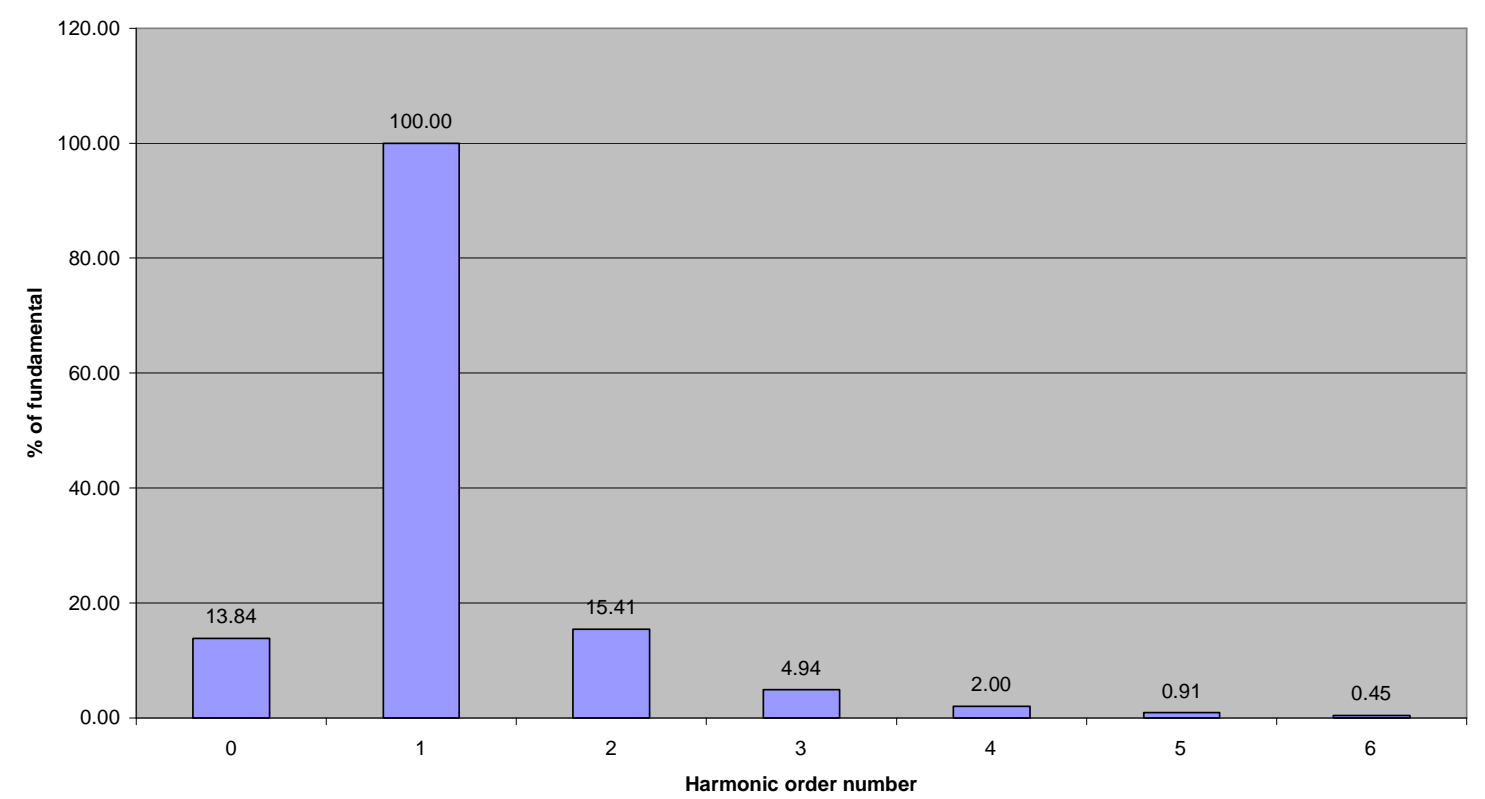

Figure 3 Frequency domain analysis of displacement waveform

Work described later in this paper suggests that somewhere between 6 and 12 harmonics are usually sufficient to give an adequate representation of the waveform. In the conventional method of extracting oil film coefficients, the fundamental components of the waveforms are used. In this example, this would give:

$A_{x x}=$ Force $/$ displacement $=0.07 / 0.0676=1.04$

However, it is known that the value of $A_{x x}$ used in the derivation of the waveforms is 1.17 (see equation 2), so this estimate is somewhat more than $10 \%$ in error. The error increases as the square of the orbit size in this region of mild non-linearity.

In summary with a sinusoidal force excitation, non-linearity causes the displacement waveform to contain an offset term and an infinite series of harmonics. It also changes the value of the coefficient associated with the fundamental frequency, leading to model errors in conventional linear methods. 


\section{The experimental identification of a higher-order dynamic oil film model}

There are three interrelated issues here. The first is the form of the mathematical model, the second is the experimental results needed to provide sufficient information for its identification and the third is the calculation method. If linearity is assumed, it is known that two suitable tests with different low-level excitations will give a result and many methods have been reported. However, almost all reported investigations lack validation of the assumption of linearity. At the start of an investigation into nonlinearity, it will not be known how many results will be needed, or what would be appropriate excitation conditions for the various tests There is very little experience/information currently available for such validation.

However, it is possible to propose a test method that can accept any reasonable initial assumption and refine it in the light of information then gathered. This new procedure can accept any reasonable oil film model and allows the researcher to choose suitable test results as the investigation proceeds. It also allows the researcher to establish when sufficient information has been acquired and allows the quality of the identification to be assessed. It is an interactive method, and the number of steps to its conclusion will not be known in advance.

A suitable calculation procedure termed 'Comprehensive Calculation Method' (CCM) to accompany it is described below. The proposed method allows the use of any two of the (i) dynamic forces, (ii) dynamic displacements and (iii) oil film model to be used to predict the third. The method uses frequency domain descriptions of the excitations and responses. The frequency domain is used because it is the most appropriate domain for repetitive signals. Repetitive (preferably approximately sinusoidal) excitations give the best possible signal/noise ratio, which is crucial to accurate identification of the dynamic oil film coefficients. The signals are represented as Fourier series, which gives a compact description.

\section{The Calculation procedure ("CCM")}

The basis of the method is to conduct a series of force balances and seek the solution giving the best overall agreement. These force balances are conveniently conducted at regular intervals round a cycle of the excitation, for each excitation (shake test) in turn. 
In the description below, it is assumed that the dynamic oil film coefficient are to be determined, so a trial set of oil film coefficients is proposed. Where displacements are to be determined from known oil film coefficients and known force excitations, a trial displacement record is proposed. Where forces are to be determined from known oil film coefficients and displacements, a trial force record is proposed. The same calculation procedure accommodates all the cases, though the last can also done by straightforward arithmetic methods.

In oil film model identification, one side of each force balance is the value from the force record, the other is the calculated force that arises from the response record and the assumed oil film coefficients. The force calculation requires both displacement (already known) and velocity, which is easily derived from the Fourier series for displacement. The force error arising from this balance is recorded. At the end of all the force balance calculations, the balance errors are assessed. In this implementation, the sum of the squares of all the errors is calculated. Other optimisation methods could be used and it is also possible to weight the balance errors according to the perceived reliability of the record in use. The assumed oil film coefficients are now iterated until the least squares solution is found.

It is most important that the Fourier series used to describe the test waveforms contain the zero-order terms. These affect the least-squares solution and must not be omitted. The zero order terms are not important in a linear system, so it is not usual to consider them. It is necessary to measure them in a higher-order investigation, so it is not possible to use high-pass filtering in the instrumentation chains. Alternative arrangements, such as fixed offsets, measurements of the zero-order terms by separate means, or very long-scale A/D conversion must be made. It is possible (but strongly deprecated) to omit measurements of the zero-order terms, and include trial values in the least squares fitting process. Simulations of this method show that the solution is much more sensitive to errors, such as due to noise on the signals, than when the zero order terms are given. 


\section{An example implementation}

All the values used in this example are non-dimensional. The dynamic forces are scaled by $W$ and the dynamic oil film coefficients are scaled by $W / c$, where $W=$ static load, $c=$ bearing radial clearance. The displacements are scaled by $\mathrm{c}$, and the velocities by $c \Omega$, where $\Omega=$ shaft angular frequency. The frequency of the excitation is defined in terms of the quantity $(\omega / \Omega)$. The shake test frequency is $\omega$ and the calculations cover one cycle of the dynamic excitation, so $\omega t$ takes values between 0 and one step less than $2 \pi$ radians $\left(360^{\circ}\right)$.

As no suitable experimental results for model identification are available, the example relates to the prediction of an orbit from known oil film coefficients and a known force record. The values used are shown below. The CCM would normally be used for oil film coefficient identification, when the order in which the items are presented would probably be different. The basic calculation procedure is identical to that for oil film coefficient identification, the only difference lies in what is iterated to give the best fit, and how many records are incorporated. In orbit prediction, only one set of force balances is used.

\subsection{Dynamic oil film coefficients}

There is only one set of assumed dynamic oil film coefficients, as the same values are used in all the calculations. In this example, the values shown in Table 1 are from Reference [2] for the first and second order coefficients for a running condition giving an eccentricity ratio of 0.6. In an oil-film model identification, these would be assumed and iterated to give the least squares solution. The trial values would normally be zero, unless better values were known. It is normally valuable to include the next highest order direct coefficients, so these are shown, though in this example these higher order coefficients are not used. The significance and use of the third order coefficients is explained later.

Table 1 (assumed dynamic oil film coefficients)

$\begin{array}{llllllll}\text { Axx } & \text { Axy } & \text { Axx' } & \text { Axy' } & \text { Axx }{ }^{2} & \text { Axy }^{2} & \text { Axx'2 } & \text { Axy'2 } \\ 1.17 & 0.30 & 1.05 & -1.65 & 4.50 & -1.58 & 4.20 & 7.95 \\ -3.6 & 3 & -1.65 & 7.2 & -3.75 & -6.45 & -6 & -12.3 \\ \text { Ayx } & \text { Ayy } & \text { Ayx' } & \text { Ayy' } & \text { Ayx }{ }^{2} & \text { Ayy }^{2} & \text { Ayx }^{\prime 2} & \text { Ayy'2 }\end{array}$




$\begin{array}{llllll}\text { Axxy } & \text { Axxx' } & \text { Axxy' } & \text { Axyx' } & \text { Axyy' } & \text { Axx'y' } \\ -4.50 & 2.40 & -11.25 & -6.75 & 7.65 & -11.70 \\ 15.75 & 2.7 & 15.75 & 12.3 & -36 & 15.75 \\ \text { Ayxy } & \text { Ayxx' } & \text { Ayxy' } & \text { Ayyx' } & \text { Ayyy' } & \text { Ayx'y' } \\ & & & & & \\ \text { Axx } & \text { Axy } & \text { Axx } & \text { Axy'3 } & & \\ 0 & 0 & 0 & 0 & & \\ 0 & 0 & 0 & 0 & & \\ \text { Ayx }^{3} & \text { Ayy }^{3} & \text { Ayx } & \text { Ayy } & & \end{array}$

\subsection{Excitation}

In this example, the oil film force is theoretical, so a very simple example has been selected, and is shown in Table 2 . The force series would normally have fewer terms than the displacement series, perhaps up to about order $(3 \omega t)$. However, in most practical examples, the force Fourier series would have more entries than those shown, as it would be normal for the excitation to be some combination of two dynamic forces in orthogonal directions, and the waveforms may not be pure sinusoids. As for the displacement data, the zero-order terms must be included. In a real oil-film model identification shake test, the oil film force is the vector difference between the excitation force and the inertia force arising from the bush mass and its acceleration, and an $(\omega / \Omega)$ ratio of 1 should be avoided for noise reasons, as discussed later.

\section{Table 2}

Fourier series for excitation forces

Excitation method $\omega / \Omega \quad$ Force

\begin{tabular}{llllll} 
& Fx & & \multicolumn{3}{c}{ Fy } \\
Const & $\operatorname{Cos}(\omega t)$ & $\operatorname{Sin}(\omega t)$ & Const & $\operatorname{Cos}(\omega t)$ & $\operatorname{Sin}(\omega t)$ \\
0.0000 & 0.0600 & 0.0000 & 0.0000 & 0.0000 & 0.0000
\end{tabular}

\subsection{Response (displacements)}

Table 3 below shows the predicted displacement orbit arising from the data above.

The displacements shown are those obtained at the conclusion of the solution process, the trial values would normally be zero.

\section{Table 3}

Fourier series for displacements

$\begin{array}{llllllllll} & \text { Const } & \operatorname{Cos}(\omega t) & \operatorname{Sin}(\omega t) & \operatorname{Cos}(2 \omega t) & \operatorname{Sin}(2 \omega t) & \operatorname{Cos}(3 \omega t) & \operatorname{Sin}(3 \omega t) & \operatorname{Cos}(4 \omega t) & \operatorname{Sin}(4 \omega t) \\ \mathbf{x} & -0.00557 & 0.068128 & 0.029633 & 0.002695 & 0.00454 & -0.00082 & 0.00062 & -0.0003 & -0.00022 \\ \mathbf{y} & -0.00492 & 0.014937 & 0.035257 & -0.00162 & 7.49 \mathrm{E}-05 & -0.00012 & -0.0004 & 8.43 \mathrm{E}-05 & -5.8 \mathrm{E}-05\end{array}$




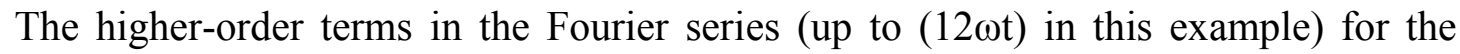
displacements are not shown and the values presented are rounded, though the values used in the calculations have the full number of digits. In an oil-film model identification, the displacement Fourier series would be measured values. Each Fourier series should contain sufficient terms to give a reasonable representation of the primary data. The displacement Series might have terms up to between $(6 \omega t)$ and $(12 \omega t)$. The zero order terms are important, normally non-zero, and must not be omitted.

\subsection{Response (velocities)}

The force balance calculations require the velocities, as well as the displacements and the oil film coefficients shown above. The velocity Fourier series associated with each displacement series is easily calculated from the Fourier series for displacement and the excitation frequency ratio $(\omega / \Omega)$, using the method described below. In the nondimensional Fourier series for one of the displacements, consider the terms of order $\mathrm{k}$. Suppose that these are:

$\left(D_{k c} * \operatorname{Cos}(k \omega t)+D_{k s} * \operatorname{Sin}(k \omega t)\right)$

the real displacement is:

$\mathrm{c}^{*}\left(D_{k c} * \operatorname{Cos}(k \omega t)+D_{k s} * \operatorname{Sin}(k \omega t)\right)$

$d / d t\left\{c *\left(D_{k c} * \operatorname{Cos}(k \omega t)+D_{k s} * \operatorname{Sin}(k \omega t)\right)\right\}=c *\left\{-k \omega * D_{k c} * \operatorname{Sin}(k \omega t)+k \omega * D_{k s} * \operatorname{Cos}(k \omega t)\right\}$

Let the corresponding terms in the non-dimensional Fourier series for velocity be:

$\left(\mathrm{V}_{k c} * \operatorname{Cos}(k \omega t)+\mathrm{V}_{k s} * \operatorname{Sin}(k \omega t)\right)$

so the real velocity is:

$\mathrm{c} \Omega *\left(\mathrm{~V}_{k c} * \operatorname{Cos}(k \omega t)+\mathrm{V}_{k s} * \operatorname{Sin}(k \omega t)\right)$

Equating terms in either $\operatorname{Cos}(k \omega t)$ or $\operatorname{Sin}(k \omega t))$ gives:

$V_{k c}=k^{*}(\omega / \Omega) * D_{k s}, V_{k s}=-k^{*}(\omega / \Omega) * D_{k c}$ 
As $k$ and $(\omega / \Omega)$ and the Fourier series for each displacement are known, the Fourier series for velocity is then calculated.

The velocities arising from the $(\omega / \Omega)$ value given in Table 2 and the displacements given in Table 3 are shown in Table 4. As for the displacement series, only the lower-order terms are shown, with their values rounded.

\section{Table 4 (calculated non-dimensional velocities)}

\begin{tabular}{llllllllll}
\multicolumn{2}{l}{ Fourier series for velocities } \\
& Const & $\cos (1 f)$ & $\sin (1 f)$ & $\cos (2 f)$ & $\sin (2 f)$ & $\cos (3 f)$ & $\sin (3 f)$ & $\cos (4 f)$ & $\sin (4 f)$ \\
xdot & 0 & 0.029633 & -0.06813 & 0.00908 & -0.00539 & 0.001859 & 0.002446 & -0.00087 & 0.001186 \\
ydot & 0 & 0.035257 & -0.01494 & 0.00015 & 0.003243 & -0.0012 & 0.000349 & -0.00023 & -0.00034
\end{tabular}

\subsection{Force balance calculations}

These are now performed, step by step. There must be more than twice as many steps as the highest order of the displacement Fourier series to avoid loss of information and/or aliasing, but it may be preferable to use considerably more, in order to give smoother output orbits etc. This allows more accurate identification of features such as maximum/minimum values and the positions at which they occur. The example in Table 5 shows $4^{\circ}$ steps, which gives 90 points per cycle, though any value over 24 steps per cycle would be adequate.

The first entry in each line of the force balance calculations is the current angular step value. The next four entries are the current values of $x, y, \dot{x}, \dot{y}$, calculated from the displacement and velocity Fourier series above, for the current angular step value. The next two entries are the oil film forces calculated from the first four entries and the assumed oil film coefficients shown above, using the equations below. As all the third order terms are taken to be zero in this example, their contribution to the force is zero, but the terms have been included for later reference.

$\mathrm{Fx}=\mathrm{Axx} * \mathrm{x}+\mathrm{Axy} * \mathrm{y}+\mathrm{Ax} \dot{x} * \dot{x}+\mathrm{Ax} \dot{y} * \dot{y}+\mathrm{Axx}^{2 *} \mathrm{x}^{2}+\mathrm{Axy} \mathrm{y}^{2 *} \mathrm{y}^{2}+\mathrm{Ax} \dot{x}^{2 *} \dot{x}^{2}+\mathrm{Ax} \dot{y}^{2 *} \dot{y}^{2}+\mathrm{Ax}$ $\mathrm{xy} * \mathrm{x} * \mathrm{y}+\mathrm{Axx} \dot{x} * \mathrm{x} * \dot{x}+\mathrm{Axx} \dot{y} * \mathrm{x} * \dot{y}+\mathrm{Ax} \dot{x} \mathrm{y}^{*} \dot{x} * \mathrm{y}+\mathrm{Ax} \dot{x} \dot{y} * \dot{x} * \dot{y}+$ $\mathrm{Axy} \dot{y} * \mathrm{y}^{*} \dot{y}+\mathrm{Axx}^{3 *} \mathrm{x}^{3}+\mathrm{Axy}^{3 *} \mathrm{y}^{3}+\mathrm{Ax} \dot{x}^{3 *} \dot{x}^{3}+\mathrm{Ax} \dot{y}^{3 *} \dot{y}^{3}$ 
$\mathrm{Fy}=\mathrm{Ayx} * \mathrm{x}+\mathrm{Ayy} * \mathrm{y}+\mathrm{Ay} \dot{x} * \dot{x}+\mathrm{Ay} \dot{y} * \dot{y}+\mathrm{Ayx}^{2 *} \mathrm{x}^{2}+\mathrm{Ayy} \mathrm{y}^{2 *} \mathrm{y}^{2}+\mathrm{Ay} \dot{x}^{2 *} \dot{x}^{2}+\mathrm{Ay} \dot{y}^{2 *} \dot{y}^{2}+$ Ayxy*x*y+Ayx $\dot{x} * \mathrm{x}^{*} \dot{x}+$ Ayx $\dot{y} * \mathrm{x}^{*} \dot{y}+\mathrm{Ay} \dot{x} \mathrm{y}^{*} \dot{x} * \mathrm{y}+\mathrm{Ay} \dot{x} \dot{y} * \dot{x} * \dot{y}+$ Ayy $\dot{y} * \mathrm{y}^{*} \dot{y}+\mathrm{Ayx}^{3 *} \mathrm{x}^{3}+\mathrm{Ayy}^{3 *} \mathrm{y}^{3}+\mathrm{Ay} \dot{x}^{3 *} \dot{x}^{3}+\mathrm{Ay} \dot{y}^{3 *} \dot{y}^{3}$

The next two entries are the known oil film forces, as derived from the values shown in the excitation specification. The last two entries show the differences between the calculated and known or measured forces.

\section{Table 5 (Force balances)}

\begin{tabular}{|c|c|c|c|c|c|c|c|c|c|}
\hline Angle & $x$ & $Y$ & Xdot & ydot & $F x$ & Fy & Target Fx Target Fy & Fx error & Fy error \\
\hline 0 & 0.06424 & 0.008377 & 0.039209 & 0.034122 & 0.059994 & $5.54 \mathrm{E}-07$ & 0.06 & $-5.9 E-06$ & $5.54 \mathrm{E}-07$ \\
\hline 4 & 0.066804 & 0.010736 & 0.034208 & 0.033435 & 0.059849 & 4.49E-06 & 0.0598540 & $-4.8 E-06$ & 4.49E-06 \\
\hline 8 & 0.06901 & 0.013042 & 0.028931 & 0.032625 & 0.059416 & 5.05E-06 & 0.0594160 & $-5.3 E-08$ & 5.05E-06 \\
\hline 12 & 0.070838 & 0.015288 & 0.023391 & 0.031693 & 0.058694 & 1.67E-06 & 0.0586890 & 5.41E-06 & 1.67E-06 \\
\hline 356 & 0.061336 & 0.005974 & 0.043923 & 0.034688 & 0.059851 & $-3.7 E-06$ & 0.0598540 & $\begin{array}{l}-3 \mathrm{E}-06 \\
\text { Error }^{2}>\end{array}$ & $\begin{array}{l}-3.7 \mathrm{E}-06 \\
4.63 \mathrm{E}-09\end{array}$ \\
\hline
\end{tabular}

This process is repeated for all the steps and the total error ${ }^{2}$ value obtained. In this example, there is only one set of force balances, in an oil film model identification, there will be a set for each shake test and the procedure is repeated for each shake test.

\subsection{Least squares solution}

The items to be iterated and the target variable are now chosen. The solution process seeks to set the target variable to the chosen value by iterations on the chosen items. The goal would be to achieve as small a target variable as possible in a reasonable number of iterations. In this example, the items to be iterated are the Fourier coefficients for the displacements, and the target variable is the sum of squares from the force balance calculations. In an oil-film identification, the items to be iterated would be the assumed oil film coefficients and the target variable would be the sum of squares of all the force balances in all the shake tests included in the calculations. As mentioned above, trial zero-order components of the Fourier series might be included in the items to be iterated. 


\section{$7 \quad$ Application of the Comprehensive Calculation Method to theoretical results}

The first application of the CCM was to predict orbits, using the 28-coefficient model given by Bannister [2].

It was intended to use these orbits as a substitute for new experimental measurements taken at excitations large enough to cause non-linear conditions. There were several matters of interest. The first was to assess the errors arising in the first order oil film coefficients in extractions by conventional methods. The second was to assess the number and nature of shake tests needed to give acceptable predictions of the known dynamic oil film coefficients and what accuracy could be expected. It was also necessary to assess how many terms would be needed in the Fourier series and how sensitive the coefficient identifications would be to noise.

Orbits were generated for three running conditions (eccentricity ratios of 0.4, 0.6 and 0.8), six excitation types ( $\mathrm{x}$-shake, y shake, forward unbalance, reverse unbalance, $+45^{\circ}$ shake, $-45^{\circ}$ shake), three excitation frequency ratios $(0.5,1,2)$ and five excitation levels (orbit sizes $2,4,6,8,10 \%$ c). This gave 270 sets of orbits, described in terms of their Fourier series. It was found that terms up to order 12 gave a good representation of the waveforms. There was no advantage in a greater number of terms. At lower excitation levels (up to what was later found to be the limit of validity of the 28-coefficient model), terms up to order 6 were sufficient.

At this stage, it was not known how much force balance error was acceptable, so values of up to a few percent of the excitation force were accepted. The first order oil film coefficients were identified from the fundamental components of the appropriate orbits (conventional shake test pairings of $\mathrm{x}, \mathrm{y}$, forward and reverse unbalance, $\pm 45^{\circ}$ ) and their associated excitations. These reconstituted coefficients were compared with the linear coefficients in the original 28-coefficient model. The results seemed generally plausible, with the errors in reconstituting the first order coefficients increasing with excitation level, excitation frequency and eccentricity ratio. It was 
later found (see below) that the results were invalid in detail, as the higher excitations lay outside the range of validity of the oil film model, so they are not presented here. Another aim was to determine how many test cases were needed to give a reliable extraction of the 28 dynamic oil film coefficients.

In a preliminary comparison, the predicted orbits for the six types of excitation were used together with the same level and excitation frequency ratio. Various combinations of level and excitation frequency ratio were considered. For each set of orbits and corresponding excitations, the 28-coefficient dynamic oil film model was identified. The identified oil film coefficients were then compared with the coefficients [2] used to generate the orbits from the known excitations. It was found that very good identifications of all the coefficients were achieved under lower excitations, but that the errors increased dramatically, particularly for the second order terms, with higher amplitude or frequency ratio results, and especially for both at once. This seemed counter-intuitive, as it would be expected that the second order oil film coefficients would be better identified at higher excitations. It seemed possible that the second order model used for generation of the orbits might be inadequate, as it was already known from the generation of a displacement waveform from a force waveform with only the first and second order coefficients for one term that the range of validity was limited. Too great a force leads to a prediction of a complex value for the displacement, which, in this context, must be a real number.

A reliable method of increasing the range of validity of the oil film model would be to increase the order of the model. However, the higher-order terms were not known. It was speculated that provisional dynamic third order oil film coefficients could be included in the model, and iterated along with the orbit coefficients to find the least squares solution. The complete set of third order oil film coefficients has 40 terms, but it was decided to use just the direct terms, of which there are 8. This gave a 36coefficient oil-film model. At lower excitations, the results were the same as previously obtained with the 28-coefficient oil-film model, but at higher excitations, the force balance errors were dramatically reduced by several orders of magnitude. The results for excitation by forward unbalance $(\varepsilon=0.6, \omega / \Omega=1)$ are shown in Fig 4. 
The errors are reported as:

(r.m.s value of force balance errors)/(r.m.s value of excitation forces)

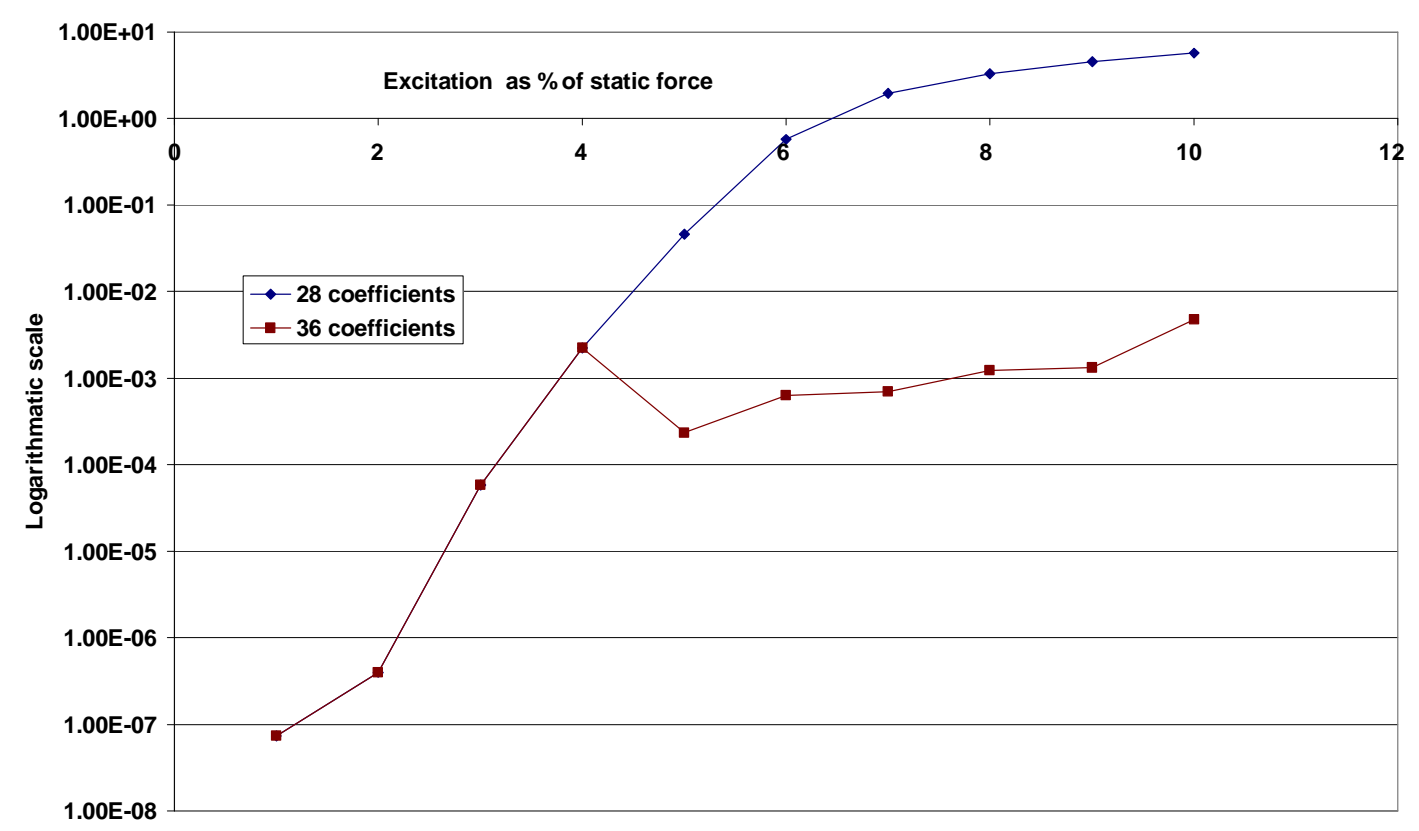

Fig 4 Force balance errors as \% of excitation force

It would seem reasonable to interpret Fig 4 as showing that the 28-coefficient model is only strictly valid in the range for which both the 28-coefficient models and 36coefficient models give the same results. In this example, this is to an excitation level of $4 \%$ of the static force for these conditions. Acceptable validity might extend $5 \%$, as the force balance errors are small (less than $0.1 \%$ of the excitation force).

The addition of the third order coefficients also had a substantial effect on the assigned values of the coefficients and the corresponding size of the orbit at higher excitations. The change in size of the orbit is illustrated in Fig 5, which compares the orbit sizes arising under the use of 8,28 and 36 oil film coefficients. 


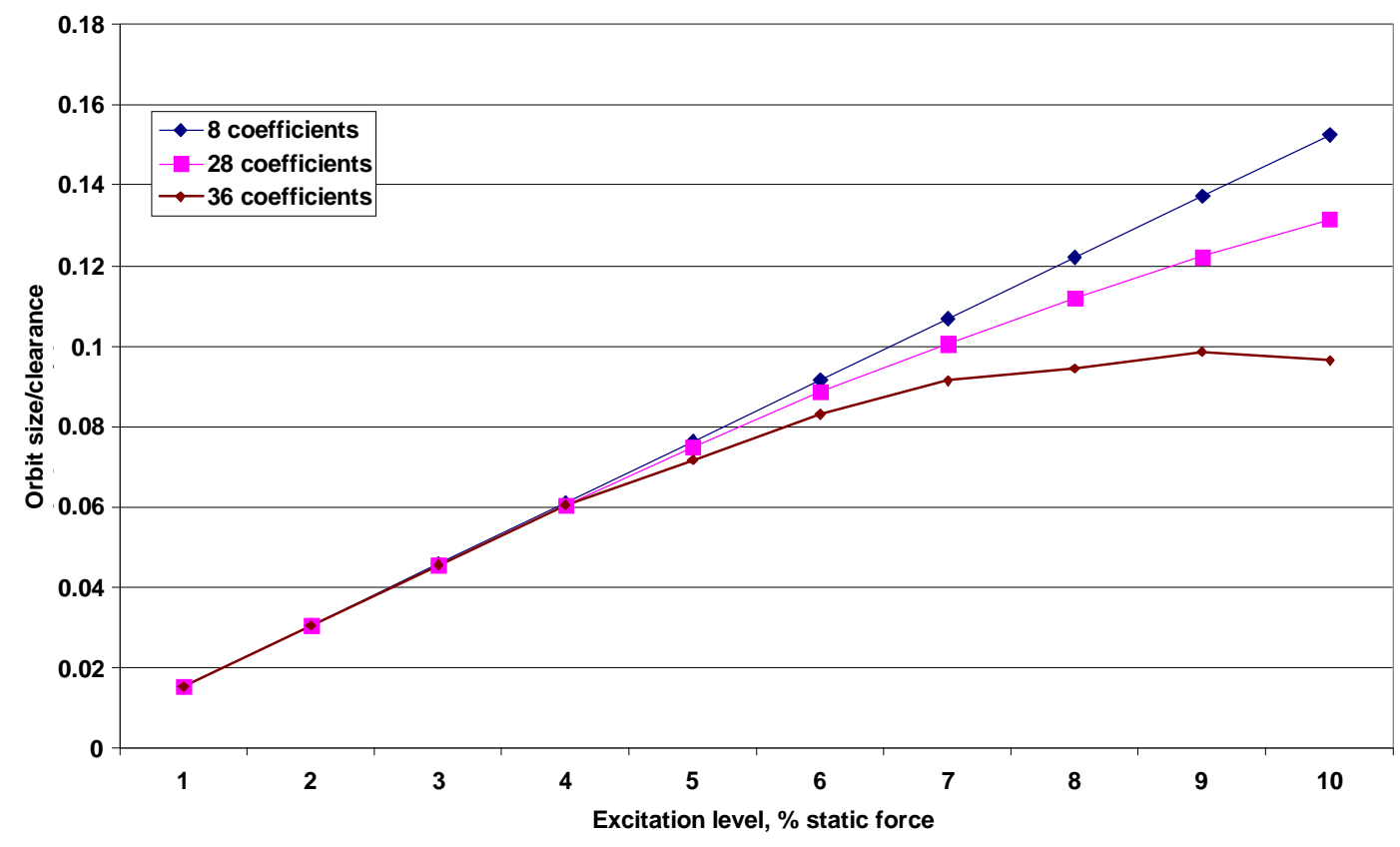

Figure 5 Orbit size vs excitation level, Forward Unbalance

It is seen that the effect of non-linearity is to reduce the size of the orbit, as already reported [2], though the shape of the curve with 36 coefficients is not entirely plausible at higher excitations. If the eight third order oil film coefficients used constituted a sufficient additional set, it would be expected that the values would be the same for all the orbit predictions. However, it was found that the values assigned were mostly rather variable, depending on the type and level of the excitation. The value for $A_{x x^{3}}$ was reasonably constant, and to a lesser degree the value for $A_{x x^{3}}$. This sensitivity to the detail of the excitation indicates that the oil film model is still inadequate.

The fitting process attempts to find the least sum of error squared, and will find incorrect values of the given coefficients to compensate for the missing forces that would have been associated with the missing coefficients. The fit error therefore does not directly represent the missing forces. However, it would be plausible to expect it to be zero if there were no missing forces. This emphasises that the least squares error has to be small for the solution to be valid. The addition of some higher-order oil film coefficients to the model is obviously not a complete answer, but it makes it possible to define the range of validity of the 28-coefficient model as up to when the third order terms start to come into play and their associated forces start to appear. This 
criterion is probably somewhat pessimistic, and slightly larger excitations may be acceptable at the price of increased identification errors.

\subsection{Range of validity of the 28-coefficient model}

Range of validity has been used above as the range over which the oil-film model makes acceptable predictions. This includes the accuracy with which the model represents the real world, but there is another issue, which affects a higher-order model, and is most easily explained by reference to a second-order model. A second order model contains terms such as shown in Equation 3 above, and the total force is that arising from all the terms noted. All the direct terms will be of the form shown and are discussed next. The key difficulty is that each quadratic equation has a limited range of values of force for which the displacement (or velocity) solution has real roots, so is mathematically limited in its range. In addition, the relationship between the contribution from each of these sets of terms and the total force depends on the circumstances, so it is not obvious what the mathematical range of validity of the full force equation night be. This is a separate problem from proper representation of the physical domain, and it is reasonable to assume that the force equation can be solved with high accuracy within its range of mathematical validity. Such a solution would have very low residual force balance error.

The mathematical range of validity of the 28-coefficient oil film model was established for the 270 test conditions described earlier and an example set of results is shown in Table 6. The range of validity is shown here in terms of orbit size, rather than excitation force. Up to the levels shown, the 28-coefficient model would be sufficient and appropriate. Other running conditions and excitation frequencies give different ranges of validity for a particular excitation type.

\section{Table 6}

\begin{tabular}{|l|l|l|l|l|l|l|}
\hline Test type & X shake & Y shake & $\begin{array}{l}\text { Forward } \\
\text { Unbalance }\end{array}$ & $\begin{array}{l}\text { Reverse } \\
\text { Unbalance }\end{array}$ & $\begin{array}{l}+45^{\circ} \\
\text { shake }\end{array}$ & $\begin{array}{l}-45^{\circ} \\
\text { shake }\end{array}$ \\
\hline $\begin{array}{l}\text { Limiting } \\
\text { Orbit size }\end{array}$ & 0.08 & 0.058 & 0.06 & 0.10 & 0.08 & 0.084 \\
\hline
\end{tabular}

All values are non-dimensional. Eccentricity ratio $=0.6$, Excitation $\omega / \Omega=1$ Orbit size is (maximum displacement)/c 
It might be observed that the ranges of validity of this second order model are less than what many would assume for a linear model $(0.1 \mathrm{c})$ !

\subsection{Number of test results needed for 28-coefficient extraction}

The number of shake test results needed to give an acceptable accuracy of extraction of the 28 coefficients was assessed by use of the excitation and response values under the conditions shown in Table 6. It would be expected that these would be optimum values for an oil film coefficient identification. For the purposes of this assessment, the results were grouped into the conventional pairs of $\mathrm{x}$ and $\mathrm{y}$ shakes, forward and reverse unbalance, and $\pm 45^{\circ}$ shakes.

A 28-coefficient oil film model was identified for various combinations of "test" results. The differences between the extracted values and the values used in the orbit generation were noted. The error was characterised as the rms value of the set of differences divided by the rms values of the set of coefficients. It was found that any single orbit and its associated excitation did not allow the extraction of the 28 coefficients, which is not surprising as the orbit is specified by 25 Fourier series terms in this example. Two orbits allowed a good identification, with less that $0.5 \%$ error in the second order terms and less than $0.005 \%$ error in the linear terms for any of the conventional excitation pairs.

Four orbits allowed even better identification, especially in the second order terms. Six orbits gave essentially zero errors $(0.0002 \%$ in the linear terms, $0.007 \%$ in the second order terms. These errors arise from shortcomings in the representation of the forces and displacements by non-infinite Fourier series and from arithmetic errors. There may have been a contribution from the generation of the orbits, but this should have been small as the range of validity of the model used was not exceeded.

In an experimental model identification there would be some (small) amount of noise as well. The sensitivity to noise was assessed by perturbation of the input data terms. The change in the identified oil film coefficients was characterised as the rms value of the set of differences divided by the rms values of the set of coefficients. Each term in the Fourier series representing the excitations and responses was perturbed by random 
amounts up to $1 \%$ of the value of the term. This was done several times, as each set of perturbations gave different errors (as would be expected). With six shake tests used, the average error in the oil film coefficients was $0.9 \%$ in the first order coefficients and $5.3 \%$ in the second order coefficients. For four tests, the average error was $1.8 \%$ for the first order coefficients and $14 \%$ for the second order coefficients, for two tests, the average error was $2.4 \%$ for the first order coefficients and $28 \%$ for the second order coefficients. It is not yet known what a reasonable estimate of noise would be, in principle, the level should be much less than the $1 \%$ assumed if suitable care is taken in signal acquisition and processing.

It is known that four linear coefficients can be identified in a single test with singlefrequency sinusoidal excitation under linear conditions. These first-order coefficients are identified from the first order displacement terms and it would seem reasonable to suppose that four second-order coefficients could also be identified from the second order displacement terms if these are present. In a similar way, it might be expected that four third-order coefficients could also be identified from the third order displacement terms if these are present. This assumes that the displacements are sinusoidal, which they are not, and that force and displacement are interchangeable, which they are not. In reality, the displacements are non-sinusoidal, and this allows more information to be extracted than for sinusoidal displacements. However, the components of the signals containing this information would be small compared with the lower-frequency components, so measurement may be difficult.

The number of shake tests needed to extract a given order of oil film model depends on the accuracy with which the waveforms can be identified, and the amount of information in the waveforms, which depends on the level and type of excitation. It would seem reasonable to assume that four tests should normally be sufficient to identify a second-order oil film model, though fewer might be sufficient under ideal conditions. More might be needed if there is much noise compared with the information. 


\subsection{Effect of neglect of non-linearity on extraction of the linear coefficients by conventional methods}

The effect is sensitive to excitation level, as well as excitation type, so it is desirable that all the assessments should be done at the same excitation level, in order to allow comparison of results. An orbit size of $5 \%$ of the bearing clearance was chosen for the first assessment, as being within the valid range for all the excitations for the excitations shown in Table 6. This is also non-small (above the 2\% limit for linearity suggested earlier). The excitation force in the $\mathrm{Y}$ shake was $0.15 \mathrm{~W}$, which was considered more than an experimenter would use in the circumstances, so this was reduced to 0.1 , giving an orbit size of $3.3 \% \mathrm{c}$ for this test only. The errors in identification of the linear coefficients were $0.6 \%$ (rms value of the set of errors/rms value of the set of coefficients) for (Fx, Fy) shakes, 1.1\% for forward and reverse unbalance and $1.9 \%$ for $\pm 45^{\circ}$ shakes. None of these errors are very large at these excitation levels for these test conditions, but they would increase at higher excitation frequencies or excitation levels, or at higher eccentricity ratios. The (Fx, Fy) shake test method gave the lowest errors, so is to be preferred.

\subsection{Use of the second-order model to extract the linear oil film coefficients}

It is possible to use a 28-coefficient extraction, perhaps with six shake tests, and only present the eight linear coefficients. This would offer very high accuracy in the absence of noise, but it is currently not known whether this is realistic. Adequate accuracy can be obtained by a conventional (Fx, Fy) test at low excitation levels (better than $0.1 \%$ error due to non-linearity at an orbit size of under $2 \% \mathrm{c}$ ). It would be useful for future investigators to compare the experimental results from the two methods.

\section{Application of the CCM to existing experimental results}

As there was no Bearing Rig available to obtain suitable new results at the time of this work, an attempt was made to use existing results. It was known that this might not be very satisfactory, as the tests had not been made with this application in mind, but it seemed valuable to assess what problems might arise in an experimental identification of higher-order oil film coefficients by this new method. 
The results selected were obtained some years ago, in a study of a high-speed journal bearing. The results seemed interesting in that the displacements contained significant amounts of harmonics, possibly allowing the identification of higher-order oil film coefficients from tests originally aimed at determining the linear oil film coefficients.

The linear dynamic oil film coefficients had been obtained by one of the variants of the method of selected orbits [5]. A figure-of-eight orbit is set up with its crossover at approximately right angles and reasonably close to the static running position. This is achieved by suitable use of both actuators at once, the one excited at twice the frequency of the other. Prior to this dynamic test, the oil film stiffness coefficients are determined statically by the method of incremental loading. The region of the crossover is studied, obtaining the crossover point, and the directions of the arms of the orbit at the crossover point. The velocities at the crossover are also obtained for each arm. The force record is known and the results are related to give the linearised oil film velocity coefficients. This allows the determination of the four velocity coefficients in one test.

There were no different types of shake tests done at any given running conditions, as the method allows the extraction of the linear oil film velocity coefficients with only one dynamic test (the stiffness coefficients are measured in a static test). However, some tests were repeated with different combinations of synchronous and twice synchronous approximately sinusoidal excitations. A suitable pair of results was selected.

The first task was to represent the time histories as Fourier series. At this stage, it was not known how many terms would be appropriate, so various orders of series were calculated with the CCM and compared with the raw data. The basis of the method is to assume a Fourier series for each of the orthogonal displacements, and work through a cycle of the data, comparing the measured values with the predicted values and doing least squares error iterations on the assumed Fourier coefficients. The number of Fourier coefficients can be altered and the process repeated. 
It is always necessary to strike a balance between too few terms in the series, when the general shape is incorrect, and too many, when the detail, which may be due to noise, is followed too closely. In this example, it was found that series to $(2 \omega t)$ and $(4 \omega t)$ did not follow the measured points adequately, but that series to $(6 \omega t)$ and $(12 \omega t)$ both gave good fits to the general shape, without following noise irregularities. Both fits were very close in shape and both gave similar fit errors, which now indicate the noise levels. It was concluded that either would be adequate. A fit to order $(12 \omega t)$ for one of the example displacements is shown in figure 6. The force record showed less scatter and a lower harmonic content.

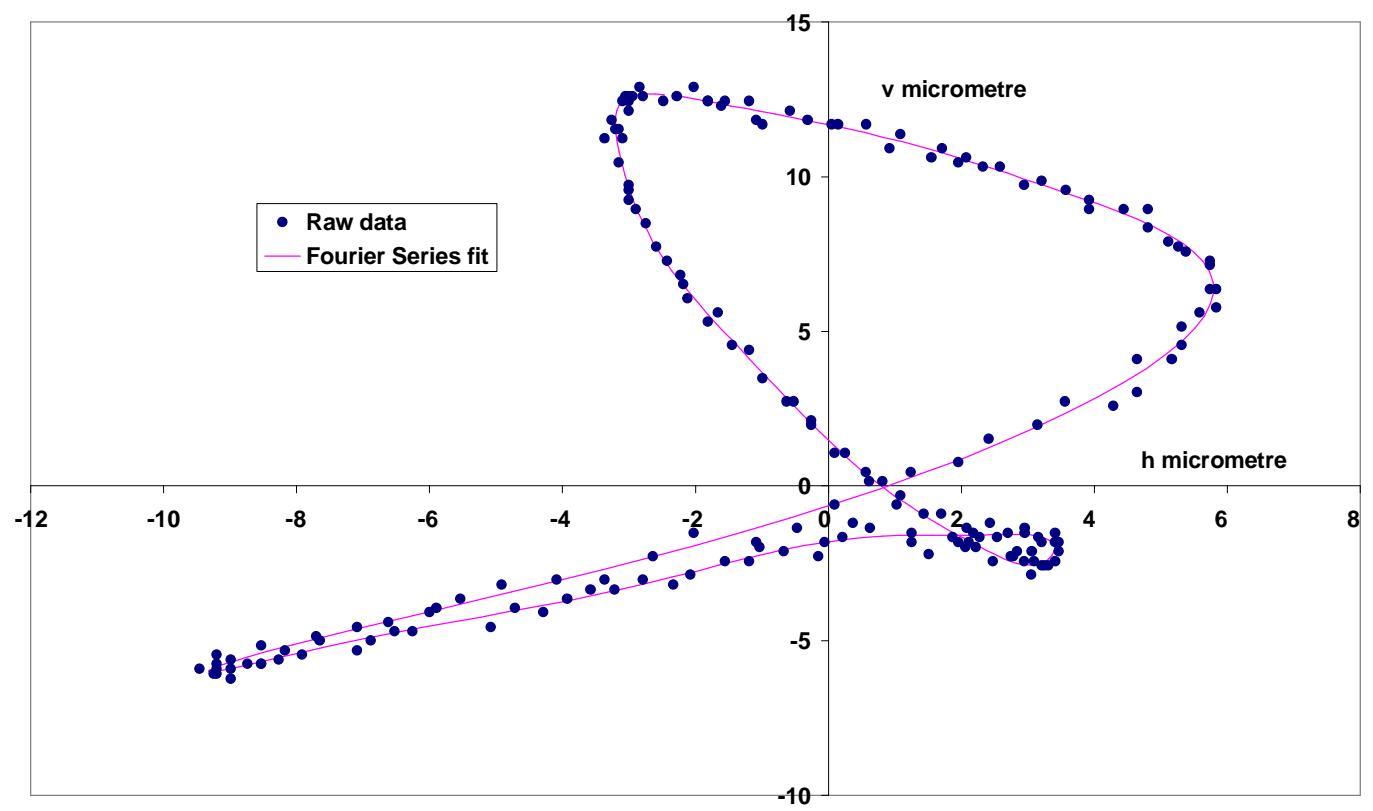

Figure 6 Comparison of raw and fitted data for a displacement orbit

The CCM was used in coefficient extraction mode for two shake tests, one with the dynamic force in the horizontal (h) direction at twice the frequency of the dynamic force in the vertical (v) force, the other with the frequencies reversed. Both the results were at approximately the same force level. On running the CCM, with oil film models of varying complexity, it was found that the force balance errors were very large, sometimes half or even more of the excitation forces. This is known to indicate a problem, as force balances should be much better than $1 \%$ if the results are to be reliable. Additionally, the 36-coefficient model indicated a large force contribution from the third order oil film conditions. 
All this indicates that the excitation levels were too high for a 28-coefficient model to cope, and a higher-order oil film model would be needed to obtain a sufficient range of validity. However, a higher-order model would need many more shake tests than were available for its identification. This, of course, means that the linear coefficients declared at the time of the tests would have a very restricted applicability. This is illustrated in Fig 7, where an orbit predicted from the declared coefficients for the known excitation is compared with the observed orbit. The axes have been rotated through $45^{\circ}$ and the displacements normalised compared with Fig 6.

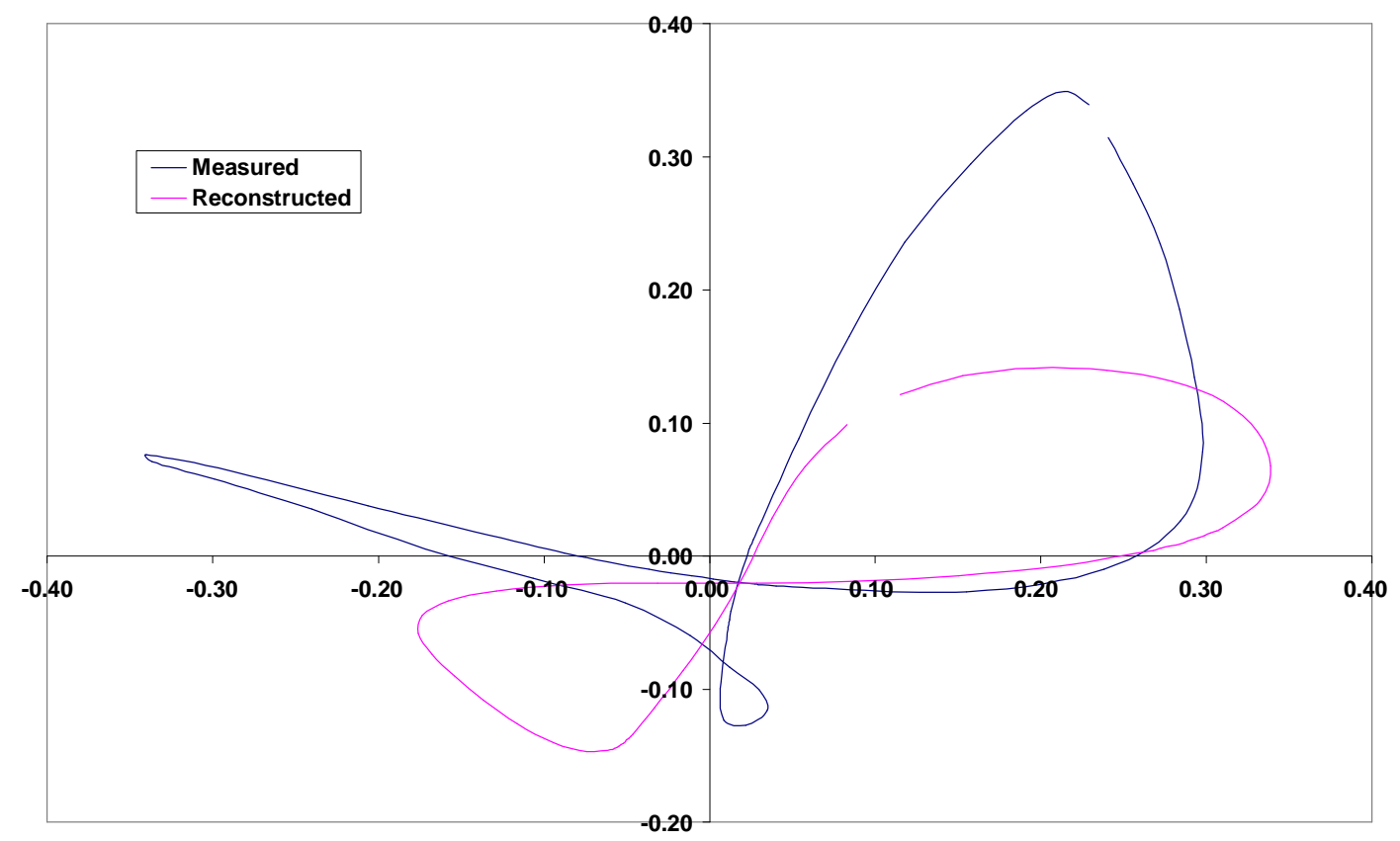

Fig 7

\section{Comparison of predicted and observed orbits}

It is seen that the orbits only agree in the region of the crossover, which is where the declared oil film coefficients were identified. This indicates that a more complete oil film model is needed if more of the working range is to be described. The discrepancy of slopes at the orbit crossover is due to an unsuitable choice of data processing in the original work, allowing a high sensitivity to noise. A rework of the method using the linearised oil-film coefficients derived from Fourier series fitted data at the crossover gave much better agreement at the crossover, though the general shape of the rest of the orbit was similar to that shown. 


\subsection{Comments on the application to existing experimental results}

This exercise illustrates those results suitable for the identification of higher-order dynamic oil film coefficients are best obtained in bespoke tests, in which the results are obtained iteratively. In this example, there were not enough different excitation conditions to allow the identification of the higher-order coefficients. All the attempted force balances led to very high errors, indicating that the model was too small. If the original aim had been this identification, it would have become apparent that more excitation conditions were necessary. These could have been done at the time of testing. However, the CCM allowed the identification of this problem, and showed that the lack of extraction of a higher-order oil film model should not be attributed to poor quality results or a poor identification procedure, but simply on an insufficient number of suitable shake test results.

\section{The measurement of the dynamic properties of a journal bearing in the non-linear region.}

It has not yet been possible to apply the new calculation method to an interactive experimental test series, so the proposals below are not validated, but are set out to encourage other workers to enter this field.

The test arrangements and the shake tests themselves would be would be very similar to those normally used for linear identifications, except that the test waveforms would be described as Fourier series (including the zero-order terms), instead of only the fundamental terms. As the zero-order (constant) terms and the harmonics would normally be quite a small proportion of the signal, and these identify the higher-order coefficients, it is most important that the dynamic results are of high accuracy.

The amount of information needed to identify a higher order model is much greater than that needed for a linear model, and it is not possible to predict how much relevant information there may be in a given shake test, so it is not possible to predict in detail what test series would be appropriate. It is therefore proposed that the test series is progressed in the light of the test results already obtained.

It is envisaged that the operator will run the calculations while the Bearing Rig is still running at the target conditions, so that alternative, additional or repeat dynamic tests 
can be run as required. The method is interactive, and the operator has various choices as the coefficient identification proceeds. The first choice for the operator is the oil film model. The method can use any reasonable oil film model and this example assumes that the operator has decided on a 36-coefficient model.

An initial set of shake tests is now conducted. It is probably reasonable to use all the excitations regarded as conventional in linear extractions, using six tests with excitations in the $\mathrm{x}$-direction, in the $\mathrm{y}$ direction, at $+45^{\circ}$ to horizontal, at $-45^{\circ}$ to horizontal, forward unbalance and reverse unbalance. It is probably reasonable to use excitation levels giving an orbit size of about 0.1 of the bearing clearance, except for the y-excitation, which would probably require too great an excitation force, so this could be set to about 0.1 of the static load. The excitation frequency must be chosen so that significant spectral lines in the shake response spectrum do not coincide with significant lines in the shaft signature, in order to avoid noise problems. It is not necessary that the same excitation frequency is used for all the shake tests, so this can be adjusted as required. The excitation frequency ratio would normally be between about 0.5 and 1 .

When the initial shake tests have been completed, the results are reviewed, most easily by using the CCM to extract the 36 coefficients and give the force fit errors. The forces associated with the third order coefficients should also be calculated. If all the force fit errors are small (less than $0.1 \%$ of the excitation level) and the third order forces are non-zero but less than about $5 \%$ of the excitation level, the results may be considered acceptable as they stand. If the force balance errors are small, and the third order forces are zero in some of the tests, it may be useful to repeat these tests at a higher excitation level. If the force balance errors are too large, and/or the third order forces are too large, the appropriate tests should be repeated at a lower excitation level. The new results should be substituted for any results at too high excitation level. This process should be repeated until the force balance errors are small enough and the third order forces nonzero, but small.

As a final check, redundant tests should be run, possibly with different frequency ratios, to give a set of results with more than six shake tests. These should not alter the values of the observed oil film coefficients. It is possible to propose other iteration strategies 
and/or error analysis methods, including error-sensitivity analyses, but each worker will have their own ideas on how to proceed.

\section{Discussion}

The measurement of the linear dynamic properties of a journal bearing is widely perceived as being rather prone to scatter and variance of the results, though it has been shown elsewhere [6], [7] that this need not be so. It has also been generally considered that taking non-linearity into account compounds the problems so much that only a very few workers have ventured into this field. This Paper has shown that this is an unnecessarily pessimistic view, and non-linearity can be tackled in a straightforward manner

For many purposes, such as predicting response to unbalance and stability boundaries in reasonably well-balanced turbo-machinery, linear dynamic oil film models are acceptable, as long as the oil film coefficients have been identified under lowexcitation conditions. Measurement made at high excitation levels but represented by a linear model will only apply under the same conditions as in the tests, so are of limited use. A past difficulty has been in validating that the test excitations were small enough for a linear model to be valid. This is now easily checked by use of the CCM (whatever the original method of identifying the coefficients) and comparing the predicted and observed responses to the excitations, preferable by comparing orbits. The data must contain all the components of the test signals, not just the fundamental components. It is then immediately obvious whether the model is adequate. An example is shown in Fig 7. It is hoped that many workers claiming linearity will make this check as a routine part of the investigation. It might be mentioned that the CCM can be used for linear oil film model identifications, when it can accept the results from more than two shake tests, so allowing a reduction in uncertainty compared with conventional methods.

At higher levels of excitation, where non-linearity starts to matter, the waveforms become non-sinusoidal. As the waveforms are normally repetitive, it is possible to represent them by Fourier series. Under excitation by sinusoidal forces, it has been shown that non-linearity changes the fundamental component of the displacement spectrum, which is why linear oil film coefficient extraction methods give errors at 
higher excitation levels. The novel calculation method described in this Paper accepts these Fourier series and permits the correct identification of the dynamic oil film coefficients. The method relates the excitations, responses and oil film model through a series of force balances, and can use any two to predict the third.

The initial application of the CCM to the prediction of orbits from given dynamic oil film coefficients and specified excitations demonstrated that this is easy within the range of validity of the oil film model, but not possible outside this range. This is because the range of validity might be limited by either by acceptable representation of the real world, or (as here) by the mathematical constraints in the model equation. There is no corresponding problem when the CCM is used for predicting the orbits from linear coefficients, or when the CCM is used in oil film coefficient identification mode. However, it is not immediately obvious what the range of validity of the model might be, as it is not a constant.

It has been found that the range of validity of the second order oil film model is much less than might be expected, less than $10 \%$ c for a typical example, which many would regard (incorrectly) as being in the linear range. However, the range of validity depends greatly on the detail of the excitation. Orbit shape is very important, but needs to be assessed for individual cases, high frequency excitation and high eccentricity ratios generally reduce the range.

A method of assessing the range of validity of the model has been developed, and is believed to be robust. The method consists of adding the eight next highest order direct coefficients to the model. The values are unknown, but are estimated as part of the solution process. The errors arising in the solution process are compared for a range of orbit sizes, when it should be found that the higher-order model has a greater range of validity, as judged by the force balance errors. Where the two error curves diverge indicates the limit for the model with the lesser number of coefficients, the limit of validity of the higher-order model is less well defined, but may be taken to be when the errors become unacceptable. This may be indicated by the shape of the error curve, as well as by the individual error values. The divergence of the models may also be identified by the onset of forces associated with the higher-order coefficients. 
The experimental identification of higher-order dynamic oil film coefficients by the new method uses experimental equipment and techniques very similar to those already widely used, so many workers could enter this field. The identification of a higher-order dynamic oil film model needs more than two shake tests, but the shake tests themselves can be similar to conventional tests, though probably at a higher excitation level. The only significant difference in signal acquisition and processing is that the test signals are represented as a Fourier series, including the zero-order terms, instead of just the fundamental component.

It has been observed that the use of sinusoidal force excitation, giving non-sinusoidal responses, although convenient and conventional, has some disadvantages. The most serious is that some of the harmonics of the displacement (an infinite series) might coincide/nearly coincide with harmonics of the shaft signature, leading to a noise problem. This can be overcome by choice of excitation frequency for the lower spectral lines, but this might need fine tuning for higher spectral lines. The second is that it is not obvious what levels of excitations should be used for a chosen oil film model (or vice-versa) as the harmonics in the responses do not directly indicate how much non-linearity is present. This leads to the need for iterative extractions, which might result in some of the initial tests being found unsuitable, and need abandonment.

Both these problems may be avoided by the use of non-sinusoidal force excitations that give substantially sinusoidal responses. Under sinusoidal responses, the harmonics in the forces extend only to the order of the non-linearity excited, which for an acceptable model size would not exceed third order. Assuming that the required excitations can be set up in a timely manner, this should give a very convenient and robust experimental technique.

It is hoped that this Paper will encourage workers using linear models to check that the assumption of linearity is valid by comparing predicted and observed orbits, and that some workers may be emboldened to consider non-linear dynamic oil film models. 


\section{References}

1. Müller-Karger, C.M., Barrett, L.E., and Flack, R.D., Influence of Fluid Film NonLinearity on the Experimental Determination of Dynamic Stiffness and Damping Coefficients for Three-Lobe Journal Bearings. Tribology Transactions, Vol. 40, No.1, 49-56. 1997

2. Bannister, R. H., A theoretical and experimental investigation illustrating the influence of non-linearity and misalignment on the eight oil film force coefficients. Proc. I Mech E Conference on Vibrations in Rotating Machinery, Cambridge, Sept 1976. Paper C219/76, 271-278. 1976

3. Parkins, D. W., Theoretical and experimental determination of the dynamic characteristics of a hydrodynamic journal bearing. ASME Journal of Lubrication Technology, Vol.101, 129-139. 1979.

4. Zhao, S.X., Dai, X. D., Meng, G., and Zhu, J. (2005), An experimental study of nonlinear oil-film forces of a journal bearing, Journal of Sound and Vibration, 2005, vol. 287 (4-5), p. 827-843

5. Parkins, D.W.,Measurement of Oil Film Journal Bearing Damping Coefficients-An Extension of the Selected Orbit Technique,ASME Journal of Tribology, Vol. 117, No. 4, 696-701, 1995.

6. G. D Wale and D. Mba, Identifying and minimising uncertainty for experimental journal bearing studies. 'International Journal of Rotating Machinery', Volume 2005, Number 3, Dec 2005. 221-231.

7. D. Wale, D Mba, A journal bearing test rig with reduced uncertainty: some design considerations, Submitted and under review. Journal of testing and evaluation, Volume 35, Issue 4, ASTM, ISSN: 0090-3973, 2007. 